\title{
POSITIVE HARMONIC MAJORIZATION OF THE REAL PART OF A HOLOMORPHIC FUNCTION
}

\author{
STEPHEN J. GARDINER
}

(Communicated by Clifford J. Earle, Jr.)

\begin{abstract}
Let $U$ be the unit disc. This paper investigates which domains $D$ in the complex plane have the property that $\mathscr{R} e f$ belongs to $h^{1}$, or the more restrictive property that $e^{f}$ belongs to the Smirnov class $\mathscr{N}^{+}$, for every holomorphic function $f: U \rightarrow D$.
\end{abstract}

\section{INTRODUCTION}

For each domain (i.e., connected open set) $D$ in $\mathbb{C}$, let $\mathscr{H}(U, D)$ be the class of all holomorphic functions from the open unit disc $U$ into $D$. As usual, let $\mathscr{N}$ be the Nevanlinna class of all holomorphic functions $f$ on $U$ for which

$$
\sup _{0<r<1} \int_{0}^{2 \pi} \log ^{+}\left|f\left(r e^{i \theta}\right)\right| d \theta<\infty .
$$

Each function $f$ in $\mathscr{N}$ has a nontangential limit, denoted by $f\left(e^{i \theta}\right)$, at almost every boundary point $e^{i \theta}$. The Smirnov class $\mathscr{N}^{+}$is the subclass of functions $f$ in $\mathscr{N}$ for which

$$
\int_{0}^{2 \pi} \log ^{+}\left|f\left(r e^{i \theta}\right)\right| d \theta \rightarrow \int_{0}^{2 \pi} \log ^{+}\left|f\left(e^{i \theta}\right)\right| d \theta \quad(r \rightarrow 1-) .
$$

A discussion of the classes $\mathscr{N}$ and $\mathscr{N}^{+}$can be found in Garnett [5, Chapter II].

Theorem A below is classical; see Helms [6, Theorem 8.33] for the "if" assertion and Frostman [4, §52] or Nevanlinna [8, VII, §4.2] for the converse. Theorem B was established more recently by Ahern and Cohn [1]. For an introduction to the notion of thin sets the reader is referred to [6, Chapter 10].

Theorem A. Let $D$ be a domain in $\mathbb{C}$. Then $f \in \mathscr{N}$ for every $f$ in $\mathscr{H}(U, D)$ if and only if $\partial D$ has positive logarithmic capacity.

Theorem B. Let $D$ be a domain in $\mathbb{C}$. Then $f \in \mathscr{N}^{+}$for every $f$ in $\mathscr{H}(U, D)$ if and only if $\mathbb{C} \backslash D$ is nonthin at $\infty$.

In this paper we investigate which domains $D$ have the property that $e^{f} \in$ $\mathscr{N}$, or that $e^{f} \in \mathscr{N}^{+}$, for every $f$ in $\mathscr{H}(U, D)$. We note that $e^{f} \in \mathcal{N}$ if

Received by the editors June 30, 1991; presented to the Society, March 21, 1992 in Springfield, Missouri.

1991 Mathematics Subject Classification. Primary 30D50; Secondary 31A15. 
and only if the real part of $f$ can be written as the difference of two positive harmonic functions on $U$, i.e., $\mathscr{R} e f \in h^{1}$. Further (assuming that $e^{f} \in \mathscr{N}$ ), $e^{f} \in \mathscr{N}^{+}$if and only if $\mathscr{R} e f$ is majorized in $U$ by the Poisson integral of its nontangential boundary values.

We will denote the right half-plane by $D_{0}$. It is obviously the case that if $D \subseteq D_{0}$ then $\mathscr{R e} f \in h^{1}$ for every $f$ in $\mathscr{H}(U, D)$. The following result describes the situation for simply connected domains that contain $D_{0}$.

Theorem 1. Let $D$ be a simply connected domain that contains $D_{0}$. Then $\mathscr{R e} f \in h^{1}$ for every $f$ in $\mathscr{H}(U, D)$ if and only if

$$
\int_{-\infty}^{\infty} \frac{\operatorname{dist}(i y, \partial D)}{1+y^{2}} d y<\infty .
$$

As will be seen in $\S 2$, Theorem 1 follows easily from a known result on the angular derivative problem. Now suppose that $D$ is a simply connected domain that contains $D_{0}$ and satisfies (1). If $D_{1}$ is a domain (not necessarily simply connected) contained in $D$, then clearly $\mathscr{R} e f \in h^{1}$ (or, equivalently, $e^{f} \in$ $\mathscr{N})$ for every $f$ in $\mathscr{H}\left(U, D_{1}\right)$. The following result identifies which of these domains have the stronger property that $e^{f} \in \mathcal{N}^{+}$for every $f \in \mathscr{H}\left(U, D_{1}\right)$.

Theorem 2. Let $D$ be a simply connected domain that contains $D_{0}$ and satisfies (1), and let $D_{1}$ be a domain contained in $D$. Then $e^{f} \in \mathcal{N}^{+}$for every $f$ in $\mathscr{H}\left(U, D_{1}\right)$ if and only if $\mathbb{R}^{4} \backslash D_{1}^{*}$ is nonthin at $\infty$, where

$$
D_{1}^{*}=\left\{\left(x_{1}, \ldots, x_{4}\right) \in \mathbb{R}^{4}:\left(x_{1}^{2}+x_{2}^{2}+x_{3}^{2}\right)^{1 / 2}+i x_{4} \in D_{1}\right\} \text {. }
$$

Here $\infty$ denotes the Alexandroff point for $\mathbb{R}^{4}$. The condition " $\mathbb{R}^{4} \backslash D_{1}^{*}$ is nonthin at $\infty$ " is equivalent to " $D_{0} \backslash D_{1}$ is not minimally thin at $\infty$ with respect to $D_{0}$ ", but the proof of Theorem 2 (see $\S 3$ ) does not use any results concerning minimally thin sets.

Let $\mathrm{PI}[g]$ denote the Poisson integral in $U$ of a function $g$ in $L^{1}(\partial U)$. The following is a simple consequence of Theorem 2 .

Corollary. Let $D_{1}$ be a domain contained in $D_{0}$. Then $u=\operatorname{PI}\left[\left.u\right|_{\partial U}\right]$ for every $f=u+i \nu$ in $\mathscr{H}\left(U, D_{1}\right)$ if and only if $\mathbb{R}^{4} \backslash D_{1}^{*}$ is nonthin at $\infty$.

\section{Proof of TheOREM 1}

2.1. The following result is due to Oikawa [9], who formulated it in terms of an infinite strip rather than a half-plane. A closely related result had previously been given by Rodin and Warschawski [10, Theorem 2].

Theorem C. Let $D$ be a simply connected domain that contains $D_{0}$. Then (1) holds if and only if there is a one-to-one conformal map $g$ of $D$ onto $D_{0}$ such that $g(z) / z$ has a finite nonzero limit as $|z| \rightarrow \infty$ in $\left\{r e^{i \theta}:|\theta|<\theta_{0}\right\}$ for each $\theta_{0}$ in $(0, \pi / 2)$.

The "if" part of Theorem 1 follows easily. To see this, let $D$ be as in the statement of Theorem 1 and suppose (1) holds. Then there is a function $g$ as in Theorem C. Let $z=x+i y$, and put $l=\lim _{x \rightarrow \infty} g(x) / x$. Clearly $l$ is real, so $l \in(0, \infty)$. Thus $\mathscr{R} e g$ is a positive harmonic function on $D$ whose Poisson integral representation in $D_{0}$ includes the term $l x$. Hence $\mathscr{R} e g$ majorizes $l x$ on $D_{0}$. It follows that if $f \in \mathscr{H}(U, D)$ the function $l^{-1} \mathscr{R} e g \circ f$ is a positive harmonic majorant of $\mathscr{R} e f$, and this implies that $\mathscr{R} e f \in h^{1}$. 
2.2. Conversely, suppose that $D$ is a simply connected domain that contains $D_{0}$ and that $\mathscr{R} e f \in h^{1}$ for every $f$ in $\mathscr{H}(U, D)$. It is certainly not the case that $\mathscr{R e} f \in h^{1}$ for every holomorphic function on $U$ (see below), so $D \neq \mathbb{C}$. Thus we can choose $f$ to be a one-to-one conformal mapping of $U$ onto $D$. Let $f=u+i \nu$. By hypothesis, $u$ has a positive harmonic majorant, $h$ say, on $U$. Since $h \circ f^{-1}(z) \geq u \circ f^{-1}(z)=x$, the positive harmonic function $H=h \circ f^{-1}$ majorizes $x$ on $D$. We define $\phi: \mathbb{R} \rightarrow$ $[0, \infty)$ by $\phi(y)=\operatorname{dist}(i y, \partial D)$. If $\phi(y)>0$ then $D$ contains the open disc of centre $i y$ and radius $\phi(y)$. Thus, applying Harnack's inequalities, we obtain $H(i y) \geq C H(\phi(y) / 2+i y) \geq C \phi(y) / 2$, where $C$ is a positive constant. Since $\int_{\{\phi(y)>0\}} H(i y) /\left(1+y^{2}\right) d y<\infty$, it is now clear that (1) holds.

\section{Proof of Theorem 2}

3.1. We recall some definitions. A positive harmonic function is called quasibounded if it can be expressed as the limit of an increasing sequence of bounded positive harmonic functions. Let $W$ be an open subset of $\mathbb{R}^{n} \quad(n \geq 2)$, let $s$ be a positive superharmonic function on $W$, and let $A \subseteq W$. Then the reduced function (or réduite) of $s$ relative to $A$ in $W$ is defined to be the infimum of all positive superharmonic functions $S$ on $W$ that satisfy $S \geq S$ on $A$. A subset $A$ of $\mathbb{R}^{n} \quad(n \geq 3)$ is said to be thin at $\infty$ if the reduced function of (the constant function) 1 relative to $A$ in $\mathbb{R}^{n}$ is less than 1 at some point of $\mathbb{R}^{n}$. The following lemma is an immediate consequence of Huber [7, Lemma].

Lemma A. Let $A \subseteq D_{0}$. The following are equivalent:

(i) the reduced function of $z \mapsto x$ relative to $A$ in $D_{0}$ equals $x$;

(ii) the set $A^{*}=\left\{\left(x_{1}, \ldots, x_{4}\right):\left(x_{1}^{2}+x_{2}^{2}+x_{3}^{2}\right)^{1 / 2}+i x_{4} \in A\right\}$ is nonthin at $\infty$.

3.2. Now let $D$ be as in the statement of Theorem 2. It follows (see $\S 2.1)$ that the subharmonic function $x^{+}$has a harmonic majorant in $D$. Let $h$ denote the least harmonic majorant of $x^{+}$in $D$.

Suppose that $\mathbb{R}^{4} \backslash D_{1}^{*}$ (and hence also $\left.\left(D_{0} \backslash D_{1}\right)^{*}\right)$ is nonthin at $\infty$. It follows from Lemma $A$ that if $s$ is any positive superharmonic function on $D$ that majorizes $h$ on $D \backslash D_{1}$ so that $s(z) \geq x$ on $D_{0} \backslash D_{1}$, then $s(z) \geq x$ on $D_{0}$. Hence $s$ is a superharmonic majorant of $x^{+}$on $D$, and so $s \geq h$ on $D$. Thus the reduced function of $h$ relative to the set $D \backslash D_{1}$ in $D$ equals $h$ itself, and so (see Doob [3, 1.VIII.10])

$$
h(z)=\int_{D \cap \partial D_{1}} h(w) d \mu_{z, D_{1}}(w)=\lim _{m \rightarrow \infty} h_{m}(z) \quad\left(z \in D_{1}\right)
$$

where

$$
h_{m}(z)=\int_{D \cap \partial D_{1}} \min \{h(w), m\} d \mu_{z, D_{1}}(w) \quad\left(z \in D_{1}\right)
$$

and $d \mu_{z, D_{1}}$ denotes harmonic measure for $D_{1}$ and $z$. Since $h$ is a positive harmonic function on $D_{1}$ that majorizes $x$ there, it follows that if $f \in$ $\mathscr{H}\left(U, D_{1}\right)$ then $h \circ f$ is a positive harmonic function on $U$ that majorizes the real part of $f$. Further, if we define $u_{m}=h_{m} \circ f+\mathscr{R} e f-h \circ f$, then each harmonic function $u_{m}$ is bounded above and so is majorized on $U$ by the Poisson integral of its (nontangential) boundary values. It follows, on letting 
$m \rightarrow \infty$, that $\mathscr{R e} f$ is majorized in $U$ by the Poisson integral of its boundary values. Thus $e^{f} \in \mathscr{N}^{+}$for every $f$ in $\mathscr{H}\left(U, D_{1}\right)$.

3.3. To prove the converse, let $f=u+i \nu$, where $f: U \rightarrow D_{1}$ is the covering map (see Ahlfors [2, Chapter 10]). If $D_{1}$ is bounded, the result is trivial. If $D_{1}$ is unbounded then

$$
\int_{0}^{2 \pi} \frac{1-|w|^{2}}{\left|e^{i \theta}-w\right|^{2}} g\left(f\left(e^{i \theta}\right)\right) \frac{d \theta}{2 \pi}=\int_{\partial D_{1}} g d \mu_{f(w), D_{1}} \quad(w \in U)
$$

for any continuous function $g$ on $\bar{D}_{1} \cup\{\infty\}$. If we put $g(z)=\min \left\{x^{+}, m /|z|\right\}$ in (2) and let $m$ tend to infinity, it follows that

$$
u^{+}(w) \leq \int_{0}^{2 \pi} \frac{1-|w|^{2}}{\left|e^{i \theta}-w\right|^{2}} u^{+}\left(e^{i \theta}\right) \frac{d \theta}{2 \pi}=\int_{\partial D_{1}} x^{+} d \mu_{f(w), D_{1}}(z) \quad(w \in U)
$$

in view of the hypothesis that $e^{f} \in \mathscr{N}^{+}$. Since $f(U)=D_{1}$, we have

$$
(\mathscr{R e} w)^{+} \leq \int_{\partial D_{1}} x^{+} d \mu_{w, D_{1}}(z) \quad\left(w \in D_{1}\right) .
$$

It follows that $x^{+}$has a quasi-bounded harmonic majorant on $D_{1}$ and hence on $D_{1} \cap D_{0}$. Thus $x$ is a quasi-bounded harmonic function on $D_{1} \cap D_{0}$, and so

$$
\mathscr{R} e w=\int_{D_{0} \cap \partial D_{1}} x d \mu_{w, D_{1} \cap D_{0}}(z) \quad\left(w \in D_{1} \cap D_{0}\right) .
$$

Hence the reduced function of $x$ relative to $D_{0} \backslash D_{1}$ in $D_{0}$ equals $x$ itself. It follows from Lemma $A$ that $\mathbb{R}^{4} \backslash D_{1}^{*}$ is nonthin at $\infty$, and this completes the proof of Theorem 2 .

\section{REFERENCES}

1. P. Ahern and W. Cohn, A geometric characterization of $\mathrm{N}^{+}$domains, Proc. Amer. Math. Soc. 88 (1983), 454-458.

2. L. V. Ahlfors, Conformal invariants, McGraw-Hill, New York, 1973.

3. J. L. Doob, Classical potential theory and its probabilistic counterpart, Springer, New York, 1984.

4. O. Frostman, Potentials d'équilibre et capacité des ensembles avec quelques applications à la théorie des fonctions, Med. Lunds Univ. Mat. Sem. 3 (1935), 1-118.

5. J. B. Garnett, Bounded analytic functions, Academic Press, New York, 1981.

6. L. L. Helms, Introduction to potential theory, Krieger, New York, 1975.

7. A. Huber, On functions subharmonic in a half-space, Trans. Amer. Math. Soc. 82 (1956), 147-159.

8. R. Nevanlinna, Analytic functions, Springer, Berlin, 1970.

9. K. Oikawa, Remarks on conformality at the boundary, Tôhoku Math. J. (2) 35 (1983), 313-319.

10. B. Rodin and S. E. Warschawski, Extremal length and univalent functions, I. The angular derivative, Math. Z. 153 (1977), 1-17.

Department of Mathematics, University College, Dublin 4, Ireland 